\title{
Confiabilidad y validez del cuestionario de apoyo social en pacientes con cáncer de Trujillo
}

\author{
Reliability and validity of the survey of social support in patients with cancer from \\ Trujillo
}

DeYVI BaCa R. ${ }^{1}$

RECIBIDO 10/12/2015, AcEPTADO 27/03/2016

\begin{abstract}
RESUMEN
La investigación tuvo como propósito analizar la validez y confiabilidad del cuestionario MOS de Apoyo Social de Sherbourne y Stewart (1991) a pacientes con cáncer de Trujillo. Estudio de tipo instrumental. La muestra estuvo conformada por 100 pacientes con diagnóstico de cáncer, mayores de 18 años y de ambos sexos. El instrumento utilizado fue el Cuestionario MOS de Apoyo Social Percibido (MOS), desarrollado por Sherbourne y Stewart (1991), y una ficha de datos diseñada para fines del estudio. Las técnicas de análisis fueron el alfa de Cronbach para la confiabilidad y el analisis factorial exploratorio (AFE) de ejes principales para la validez con rotación Promáx. Se logró un nivel elevado de confiabilidad $(\alpha=.94)$ y un modelo de tres factores (54.240\% de la varianza total): informacional, afectivo e instrumental. El estudio concluyó en un adecuado nivel de confiabilidad y validez del instrumento, y se recomienda ampliar la población de estudio tanto con muestras clínicas (con cáncer) como no clínicas (sin cáncer).
\end{abstract}

Palabras clave: Apoyo social, cáncer,confiabilidad, validez.

\begin{abstract}
The research was aimed analyze the validity and reliability of the MOS Social Support Sherbourne and Stewart (1991) to patients with cancer in Trujillo. Study instrumental. The sample consisted of 100 patients diagnosed with cancer, aged 18 and both sexes. The instrument used was the Questionnaire MOS Perceived Social Support (MOS), developed by Sherbourne and Stewart (1991), and a data sheet designed for the study purposes. The techniques used were Cronbach's alpha for reliability and exploratory factor analysis (EFA) of principal axes for validity. The results achieved a high level of reliability $(\alpha=.94)$ and a model three factors (54.24\% of the total variance): informational, emotional and instrumental. The study concluded adequate reliability and validity of the instrument level, and recommends further study population with both clinical samples (cancer) and non-clinical (no cancer).
\end{abstract}

Keywords: Social support, cancer, reliability, validity.

1 Docente de la Facultad de Psicología y Trabajo Social de la UIGV. Correo electrónico: d.baca@uigv.edu.pe 


\section{INTRODUCCIÓN}

El apoyo social es considerado un elemento importante en el estudio del comportamiento humano que se manifiesta en la interacción social a nivel emocional, tangible o instrumental e informacional (Barrón, 1996; Schaefer, Coyne \& Lazarus, 1981). Así, sus diversas formas de expresarse permiten enfrentar una situación de enfermedad (Nieto Munuera, Abad Mateo, Esteban \& Tejeira, 2004). El apoyo social ha sido estudiado y definido teóricamente sobre la base del efecto que tiene el ambiente sobre el sujeto, asumiéndose para el presente estudio como una definición consensuada planteada por Lin, Dean y Ensel (1986; como se citó en López \& Chacón, 1999: 185): "provisiones instrumentales o expresivas, reales o percibidas, dadas por la comunidad, redes sociales y amigos íntimos".

Se distinguen dos tipos de abordaje en función de la perspectiva desde la que la estudia (Riquelme, 1997):

a. Desde una perspectiva estructural, se hace referencia a las características objetivas de la red de apoyo social, como a las características de los contactos que tienen lugar dentro de ella.

b. Desde una perspectiva funcional, se analizan los efectos o consecuencias que le suceden al sujeto y la conservación de las relaciones sociales que tiene en su red (Schaefer, Coyne \& Lazarus, 1981). Los tipos del apoyo social que se han venido reflejando en la mayor parte de las clasificaciones son emocional, tangible o instrumental e informacional (Barrón, 1996; Schaefer et al., 1981).

Otra forma de clasificar el apoyo social, según Caplan (1974; como se citó en Rodríguez, 2011), es la siguiente:

a. Apoyo social objetivo, que serían los recursos, provisiones o transacciones reales a los que la persona puede recurrir en el caso de necesitarlos.

b. Apoyo social percibido, que destaca la dimensión evaluativa que lleva a cabo la persona acerca de la ayuda con la que cree contar.

Sherbourne y Stewart (1991) consideran que las diversas investigaciones han estudiado el apoyo social o, bien, desde una perspectiva funcional o, bien, desde una perspectiva estructural; ambas líneas de investigación reportaron propiedades psicométricas poco fehacientes. Sin embargo, la propuesta de dichos autores fue diseñar un instrumento que permite evaluar de forma multidimensional el apoyo social analizando las percepciones de las personas por ser consideradas medidas más estables e importantes.

De otra parte, la prevalencia del cáncer en nuestro país está en aumento. Según las cifras oficiales del Ministerio de Salud es la segunda causa de muerte (Ministerio de Salud [MINSA], 2006). Además de ello, el impacto psicológico en la persona que lo padece puede analizarse desde múltiples esferas como son las asociadas con la enfermedad: la fase de intervención, el tipo de cáncer y el tratamiento prescrito; con el enfermo: tipo de personalidad; y con el marco social: el apoyo social y las actitudes sociales hacia la enfermedad (Cruzado, 2010; Nieto Munuera et al., 2004). 
Es relevante contar con instrumentos que evalúen el constructo apoyo social en pacientes con una enfermedad crónica, particularmente, el cáncer. Terol, López, Neipp, Rodríguez, Pastor \& Martin-Aragon (2004) señalan una amplia diversidad de instrumentos referidos al tema. Se consideró pertinente el uso del instrumento de apoyo social desarrollado por Sherbourne y Stewart (1991), denominado Cuestionario MOS de Apoyo Social (The MOS Social Support Survey), ya que fue creado en un contexto hospitalario, asumiendo una muestra de pacientes con enfermedad crónica. Dicho instrumento ha sido sometido a diversas adaptaciones internacionales, que se pasan a detallar:

Costa, Salamero y Gil (2007) realizaron una adaptación de la versión inglesa The MOS Social Support Survey, a España con una muestra de pacientes con cáncer. El instrumento conservó sus 19 ítems iniciales agrupados en tres dimensiones (apoyo emocional/informacional, apoyo afectivo y apoyo instrumental). La confiabilidad del instrumento osciló de .75 a .94 en sus dimensiones. El análisis de validez utilizado fue el AFE,obteniéndose un $65.44 \%$ de varianza explicada del constructo apoyo social. En EEUU, la versión inglesa es adaptada a un formato reducido por Moser, Stuck, Silliam, Ganzy Clough-Gorr(2012) para pacientes con cáncer de mama. El instrumento quedó compuesto por ocho ítems agrupados en dos dimensiones (apoyo emocional y apoyo tangible). La confiabilidad del instrumento osciló entre .88 y .97 . La validez se estableció por medio de correlaciones interdimensiones, oscilando entre .50 y .83 .

En Sudamérica, también se han hecho aportes significativos. Revilla, Luna, Bailón y Medina (2005, Argentina) desarrollan una adaptación del instrumento en versión inglesa a pacientes con enfermedad crónica con los 19 ítems originales en tres dimensiones (apoyo emocional/informacional, interacción afectiva positiva y apoyo instrumental). La confiabilidad calculada de las dimensiones fue de .87 a .94. Se utilizó el AFE para determinar la validez del instrumento, hallándose un $68.72 \%$ de explicación del constructo. De otro lado, Rodríguez y Enrique (2007) realizaron la adaptación de la versión inglesa con los 19 ítems agrupados en tres dimensiones (apoyo emocional/informacional, interacción afectiva positiva y apoyo instrumental). La confiabilidad de la escala total es .91 y de las dimensiones de .80 a .87. La validez hallada por medio del AFE fue $59.86 \%$ de explicación. En Brasil, se realizó una adaptación de la versión inglesa a cargo de Alonso, Montes, Menendez y Graça (2007), a pacientes con enfermedad crónica. El número de ítems se conserva y se agrupa en cuatro factores (apoyo emocional, apoyo tangible, interacción afectiva positiva y apoyo afectivo). La confiabilidad obtenida de la escala total fue .86 a .97 y de las dimensiones, .87 a .96 . La validez apostada por AFE fue $68.72 \%$ de varianza explicada. Finalmente, en un estudio realizado en Colombia por Londoño et al. (2012) bajo una muestra no clínica (personas sin cáncer). Se mantienen los 19 ítems en cuatro dimensiones (apoyo emocional/informacional, apoyo instrumental, interacción positiva y apoyo afectivo). La confiabilidad en las dimensiones osciló entre .74 y .92. La validez se estableció por medio de análisis factorial confirmatorio (AFC). 
Los estudios sudamericanos presentan niveles de confiabilidad y evidencias de validez aceptables. Empero, las diferencias en las adaptaciones realizadas están dadas por el número y tipo de dimensiones que conforman el constructo de apoyo social, al igual que las adaptaciones en España y EE.UU. Además, los métodos de análisis factorial desarrollados han sido principalmente de análisis factorial exploratorio, con excepción del estudio de Londoño et al. (2012), lo cual sugiere la dificultad de encontrar una estructura factorial definida, principalmente por la agrupación de los ítems en cada una de las dimensiones. Incluso dos estudios (Rodríguez \& Enrique, 2007; Londoño et al., 2012) han sido desarrollados sobre muestras no clínicas (personas sin cáncer).

Por lo mencionado, hay una necesidad de contar con un instrumento válido y confiable en Perú que evalúe el constructo de apoyo social, frente a la carencia de uniformidad en las adaptaciones realizadas en otros contextos y determinar qué dimensiones conforman dicho constructo. El propósito de la presente investigación fue determinar la confiabilidad de consistencia interna y validez de constructo del Cuestionario MOS de Apoyo Social de Sherbourne y Stewart (1991) en pacientes con cáncer que acuden a un hospital de Trujillo. Cabe destacar que el instrumento en adelante se denominará Cuestionario MOS de Apoyo Social Percibido por analizarse la percepción que tiene el propio sujeto sobre la cantidad y calidad de apoyo social recibido. La hipótesis de investigación enuncia que la versión adaptada del Cuestionario MOS de Apoyo Social Percibido es válida y confiable en pacientes con cáncer que acuden a un hospital de Trujillo. El presente estudio busca contribuir en la práctica clínica del psicólogo, proveyendo de un instrumento novedoso en nuestro medio, válido a nivel internacional. Se analizará si el cuestionario MOS de Apoyo Social es válido y confiable en nuestro medio y con una población de pacientes con cáncer.

\section{MÉTODO}

Según Sánchez y Reyes (2006), el presente estudio es de tipo tecnológico, ya que pretende aportar con un instrumento válido para posteriores investigaciones.

\section{Participantes}

Los pacientes con diagnóstico de cáncer (muestra clínica), mayores de 18 años y de ambos sexos, que acudieron al hospital de Trujillo durante el período noviembrediciembre del 2012. El muestreo fue intencional, de tipo no-probabilístico, ascendiendo la muestra a 100 pacientes. La muestra conformada por $22 \%$ varones y $78 \%$ mujeres. De acuerdo al grupo etario, el $6 \%$ era adulto joven (18 a 29 años), $42 \%$ fue adulto (30 a 49 años) y el $52 \%$ era adulto mayor (60 a 84 años). Asimismo, el nivel de instrucción mayoritario en la muestra fue de nivel superior (42\%), seguido del nivel secundario (30\%), nivel primario (16\%), técnico-superior (6\%) y sin nivel (6\%). En cuanto a su procedencia, se detectó que el 59\% provenía de la misma ciudad de Trujillo, mientras que un $18 \%$ lo hacía de las provincias del departamento de La Libertad y un 23\% provenía de otros departamentos (Áncash, Lambayeque y Cajamarca). 


\section{Instrumento}

El Cuestionario MOS de Apoyo Social Percibido (MOS), desarrollado por Sherbourne y Stewart (1991). Cuestionario autoadministrado de 20 ítems, conformado por dos áreas: apoyo social estructural (un ítem de respuesta abierta) y apoyo social funcional (19 ítems de respuesta múltiple); y cuatro factores: apoyo emocional/ informacional, tangible o instrumental, interacción positiva y afectivo. Evalúa, por un lado, características cuantitativas u objetivas de la red de apoyo social, tales como tamaño y densidad (ítem 1); y, por el otro, los efectos o consecuencias que le brindan al sujeto el acceso y conservación de las relaciones sociales que tiene en su red (ítems 2 a 20). Está dirigido para personas mayores de 18 años (ver Anexo 2).

Confiabilidad: Sherbourne y Stewart (1991) encontraron, a nivel de la escala general, un coeficiente alfa de Cronbach de .97; y, a nivel de las dimensiones, entre .91 y .96; niveles considerados muy adecuados. Los estudios recopilados determinarón por medio del alfa de Cronbach: a nivel de escala general, de .86 a .97; a nivel de dimensiones, de .74 a .96 .

Validez: Sherbourne y Stewart (1991) detectaron correlaciones entre las dimensiones de .72 a .88 , considerados niveles altos de correlacion. Los diversos estudios citados determinaron a través del AFE niveles entre 59\% y $69 \%$ de la varianza explicada.

\section{Recopilación y análisis de información}

Se hicieron las coordinaciones previas con la institución y servicio de oncología, posteriormente se explicó a los pacientes y, de encontrarse acompañados, a los familiares sobre el objetivo del estudio y las actividades que se pretendía realizar. Todo lo cual estuvo explicitado en el consentimiento informado que se pidió que firmen si no tenían ningún inconveniente en su participación en el estudio. Los cuestionarios fueron vaciados a una base de datos en el programa SPSS versión 21. Dentro de las pruebas estadísticas utilizadas, se puede señalar que para el análisis de la fiabilidad de consistencia interna se utilizó el alfa de Cronbach. Respecto del análisis de la validez de constructo, al no existir unicidad de la estructura factorial en las adaptaciones desarrolladas en otros países, y ante la inconsistencia de la agrupación factorial (número de dimensiones y el tipo de dimensión), se consideró un AFE concebido como un proceso de obtener un mínimo de factores que sinteticen la información aportada por un amplio número de variables (Cea, 2004). Finalmente, se utilizó el coeficiente de correlación rho de Spearman al tener una distribución no normal de las variables (ítems), ver Anexo 1. 


\section{RESULTADOS}

\section{Análisis psicométrico}

En la Tabla 1, se presentan las correlaciones ítem-test por encima de .200; por tanto, todos los ítems lograron índices de discriminación aceptables (Ary, Jacobs \& Razavieh, 1990; como se citó en Aliaga, Rodríguez, Ponce, Frisancho \& Enríquez, 2006).

Tabla 1. Correlaciones ítem - test del Cuestionario MOS de Apoyo Social Percibido ( $N=100)$

\begin{tabular}{cccc}
\hline Ítems & $\begin{array}{c}\text { Correlación elemento-total } \\
\text { corregida }\end{array}$ & Ítems & $\begin{array}{c}\text { Correlación elemento-total } \\
\text { corregida }\end{array}$ \\
\hline Ítem 2 & .438 & Ítem 12 & .576 \\
Ítem 3 & .680 & Ítem 13 & .756 \\
Ítem 4 & .565 & Ítem 14 & .668 \\
Ítem 5 & .476 & Ítem 15 & .520 \\
Ítem 6 & .677 & Ítem 16 & .550 \\
Ítem 7 & .595 & Ítem 17 & .694 \\
Ítem 8 & .718 & Ítem 18 & .716 \\
Ítem 9 & .673 & Ítem 19 & .733 \\
Ítem 10 & .707 & Ítem 20 & .634 \\
Ítem 11 & .682 & & \\
\hline
\end{tabular}

El cuestionario MOS de Apoyo Social Percibido es una escala de tipo Likert (Kerlinger\& Lee, 2002) y con altas correlaciones ítem-ítem (Welch\& Comer, 1988) entre .24 y .53; por tanto, se hizo uso de un análisis de consistencia interna a través del coeficiente alfa de Cronbach. El análisis realizado halló un alfa de Cronbach -en los 19 ítems- de .94, lo que indica un nivel elevado de consistencia interna entre los ítems y el test total (Murphy \&Davishofer; como se citó en Hogan, 2004). El procedimiento de análisis de confiabilidad es recomendable antes del análisis de validez de un instrumento a ser validado (Celina \& Campo, 2005).

\section{Evidencia de validez de constructo}

Se consideró apropiado el uso del análisis factorial exploratorio por cuanto no existe un consenso sobre las dimensiones que componen el constructo apoyo social percibido en las adaptaciones previas; e, incluso, las dimensiones no se conforman por los mismos ítems. Además permite ver si las variables se ajustan a un modelo matemático en que las variaciones de muestreo se hacen más notorias, donde se aprecian varios modelos factoriales (Reise, Waller \& Comrey, 2000); de igual modo, el tamaño muestral (100) es considerado adecuado para el AFE (Cea, 2004). Asimismo, con el objetivo de valorar la pertinencia del uso del análisis factorial, se analizaron las medidas de adecuación muestral: el valor del determinante fue de .000000466 indicando altas intercorrelaciones entre las variables; la medida 
de la adecuación de muestreo KMO fue de .887 considerada moderada, con lo cual la factorización es apropiada; y el test de esfericidad de Bartlet tuvo un valor Chi-cuadrado estadísticamente muy significativo $(\mathrm{p}<.01)$, con lo cual la matriz de correlaciones no es una matriz de identidad. Por lo anterior, se concluyó lo apropiado del análisis factorial.

Se aplicó la extracción de factores con el método de componentes principales, considerado apropiado cuando las variables (ítems) poseen distribución no normal (Costello \& Osborne, 2005). El criterio de retener factores que expliquen aproximadamente el 60\% de la varianza total (Hair et al., 1992, 1999; como se citó en Cea, 2004), encontrándose en la Tabla 2 que el modelo de tres factores explicó $54.240 \%$ de la varianza total; lo cual supone la suma de las comunalidades iniciales de cada ítem (Cea, 2004).

Para interpretar los factores con respecto a cada uno de los ítems, se eligió la rotación Promax de tipo oblicua (Hendrickson \& White, 1964; como se citó en Merino, Pflucker \& Riaño-Hernández, 2012). En la Tabla 1, la estructura factorial de tres factores se configuró de la siguiente manera: Apoyo informacional (F1), con nueve ítems; Apoyo afectivo (F2), seis ítems; y Apoyo instrumental (F3), cuatro ítems. Las saturaciones o cargas de estos fueron próximos o superiores a .50, con excepción del ítem 2 que se aproxima; por tanto, propician una clara definición e interpretación de los factores (Comrey, 1985; como se citó en Aliaga et al., 2006). Con respecto a las comunalidades (h2), concebido como proporción de la varianza total que es varianza del factor común (Kerlinger \& Lee, 2002), se encontraron valores próximos o superiores a .50 con excepción de los ítems 2 y 5 , pese a ello se conservaron al poseer adecuados niveles de cargas factoriales.

Tabla 2. Estructura factorial con rotación Promax del Cuestionario MOS de Apoyo Social Percibido Funcional $(\mathrm{N}=100)$

\begin{tabular}{ccccccl}
\hline Ítems & $\mathrm{M}$ & $\mathrm{DE}$ & $\mathrm{F} 1$ & $\mathrm{~F} 2$ & $\mathrm{~F} 3$ & $\mathrm{~h} 2$ \\
\hline Ítem 3 & 4.21 & .856 & $.532(.678)$ & $.104(.557)$ & $.205(.591)$ & .497 \\
Ítem 4 & 4.11 & .952 & $.683(.624)$ & $-.192(.382)$ & $.191(.512)$ & .404 \\
Ítem 8 & 4.16 & .907 & $.383(.654)$ & $.219(.632)$ & $.316(.702)$ & .573 \\
Ítem 9 & 4.05 & .925 & $.874(.802)$ & $-.017(.478)$ & $-.098(.497)$ & .652 \\
Ítem 13 & 4.17 & .900 & $.717(.808)$ & $.282(.648)$ & $-.120(.559)$ & .684 \\
Ítem 14 & 4.15 & .880 & $.607(.679)$ & $-.116(.476)$ & $.335(.674)$ & .540 \\
Ítem 16 & 3.96 & .931 & $.890(.673)$ & $.112(.398)$ & $-.419(.365)$ & .475 \\
Ítem 17 & 3.97 & .937 & $.831(.786)$ & $-.084(.475)$ & $.065(.626)$ & .635 \\
Ítem 19 & 4.08 & .861 & $.779(.802)$ & $.015(.539)$ & $.062(.624)$ & .651 \\
Ítem 6 & 4.50 & .759 & $.049(.542)$ & $.749(.815)$ & $.093(.512)$ & .665 \\
Ítem 10 & 4.41 & .854 & $.294(.629)$ & $.666(.770)$ & $-.075(.532)$ & .624 \\
Ítem 11 & 4.34 & .855 & $.296(.600)$ & $.414(.663)$ & $.164(.613)$ & .515
\end{tabular}




\begin{tabular}{|c|c|c|c|c|c|c|}
\hline Ítem 12 & 4.44 & .783 & $-.117(.423)$ & $.785(.716)$ & $.123(.463)$ & .517 \\
\hline Ítem 15 & 4.29 & .868 & $-.065(.403)$ & $.823(.659)$ & $-.060(.355)$ & .441 \\
\hline Ítem 20 & 4.58 & .699 & $-.022(.493)$ & $.746(.777)$ & $.132(.496)$ & .604 \\
\hline Ítem 2 & 4.35 & .880 & $-.124(.334)$ & $.288(.461)$ & $.488(.415)$ & .239 \\
\hline Ítem 5 & 4.31 & .849 & $-.257(.331)$ & $.266(.519)$ & $.737(.522)$ & .355 \\
\hline Ítem 7 & 4.30 & .859 & $.250(.521)$ & $-.167(.463)$ & $.751(.780)$ & .611 \\
\hline Ítem 18 & 4.25 & .833 & $.490(.682)$ & $-.005(.555)$ & $.424(.762)$ & .624 \\
\hline Autovalor & & & 8.899 & 1.696 & 1.058 & \\
\hline $\begin{array}{c}\% \text { Varianza } \\
\text { total }\end{array}$ & & & 44.564 & 6.510 & 3.165 & \\
\hline \multicolumn{7}{|l|}{ Correlación } \\
\hline $\mathrm{F} 1$ & & & 1 & $.646 * *$ & $.587 * *$ & \\
\hline $\mathrm{d}$ de Cohen & & & & 1.7 & 1.4 & \\
\hline $\mathrm{F} 2$ & & & & 1 & $.682 * *$ & \\
\hline $\mathrm{d}$ de Cohen & & & & & 1.9 & \\
\hline F3 & & & & & 1 & \\
\hline
\end{tabular}

Nota: $\mathrm{DE}=$ Desviación estándar; $* * \mathrm{p}<.01$; valores entre paréntesis indican la carga factorial del ítem después de la rotación Promáx.

En la Tabla 3 se hallaron correlaciones significativas que oscilaban entre $.231 \mathrm{y}$ .498, considerado un nivel de tipo medio positivo (Cohen 1988; como se citó en Nolan \& Heinzen, 2011). Ello implica que los constructos de apoyo social percibido funcional y apoyo social percibido estructural están relacionados.

Tabla 3. Correlaciones entre el apoyo social percibido estructural con el apoyo social funcional y sus factores: apoyo informacional, afectivo e instrumental $(\mathrm{N}=100)$

\begin{tabular}{cccccc}
\hline & & $\begin{array}{c}\text { Apoyo social } \\
\text { percibido funcional }\end{array}$ & $\begin{array}{c}\text { Apoyo } \\
\text { informacional }\end{array}$ & $\begin{array}{c}\text { Apoyo } \\
\text { afectivo }\end{array}$ & $\begin{array}{c}\text { Apoyo } \\
\text { instrumental }\end{array}$ \\
\hline $\begin{array}{c}\text { Apoyo social } \\
\text { estructural }\end{array}$ & Rho & $.365^{* *}$ & $.231^{*}$ & $.468^{* *}$ & $.498^{* *}$ \\
\hline
\end{tabular}

** Al nivel .01 (bilateral); * Al nivel .05 (bilateral).

\section{Análisis de fiabilidad de consistencia interna a nivel de las dimensiones}

$\mathrm{Al}$ determinarse los tres factores en el instrumento, se analizó la confiabilidad por consistencia interna de los mismos. Apreciándose en la tabla 4 niveles bajo (apoyo instrumental), moderado (apoyo afectivo) y alto (apoyo informacional) de consistencia interna entre los ítems y el test total (Murphy \& Davishofer; como se citó en Hogan, 2004). 
Tabla 4. Análisis de fiabilidad del Cuestionario MOS de Apoyo Social Percibido (N=100)

\begin{tabular}{cccc}
\hline & Apoyo informacional & Apoyo afectivo & Apoyo instrumental \\
\hline Alfa de Cronbach & .908 & .871 & .708 \\
Número de ítems & 9 & 6 & 4 \\
\hline
\end{tabular}

\section{DISCUSIÓN}

El presente estudio fue desarrollado en una muestra total de 100 pacientes con diagnóstico de cáncer que acudían a un hospital de Trujillo, cuyas edades estaban entre 18 y 84 años. El propósito fue establecer la confiabilidad de consistencia interna y validez de constructo del cuestionario MOS de Apoyo Social de Sherbourne y Stewart (1991) aplicado a tales pacientes.

En relación con la confiabilidad del cuestionario MOS de Apoyo Social, se encontró un nivel elevado de consistencia interna. Ello revela que el instrumento en mención mide con precisión y de forma consistente el constructo de apoyo social percibido (Kerlinger \& Lee, 2002). Lo referido concuerda con el estudio original de Sherbourne y Stewart (1991); al igual que Mosser et al. (2012), este último es una versión resumida. A nivel de Sudamérica, también coincide con Rodríguez y Enrique (2007) y Alonso et al. (2007). Por tanto, el instrumento a nivel internacional y local muestra niveles elevados de confiabilidad que dan mayor utilidad al mismo.

De otro lado, analizando la literatura existente para determinar la validez de constructo del instrumento por análisis factorial, se determinó el uso del AFE. El modelo más apropiado fue de tres factores que explicó un $54.240 \%$ de la varianza total. Cabe destacar que Sherbourne y Stewart (1991), al elaborar el cuestionario MOS de Apoyo Social utilizaron un Análisis Multirrasgo Multimétodo (MRMM) de la literatura previa y determinaron cinco dimensiones (apoyo emocional, informacional, afectivo, tangible y de interacción positiva); luego aplicaron un AFC donde se confirmaron resultados similares con correlaciones interdimensiones de .72 a .88 . Dichos niveles son similares a los logrados para el presente estudio, pese a que el número de dimensiones era mucho menor que en el estudio original. Así también, hay investigaciones que reportan análisis de validez por AFE con tres dimensiones con niveles próximos o superiores al $60 \%$ de explicación de la varianza total: Costa et al. (2007), 65.44\%; Revilla et al. (2005), 68.72\%; Rodríguez y Enrique (2007), 59.86\%. Todos con los mismos rótulos a las dimensiones halladas. En contraste, Alonso et al. (2012) reportaron un $72.71 \%$ de la varianza explicada con cuatro factores; mientras que Londoño et al. (2013) determinaron por AFC la presencia de cuatro factores. Esta incongruencia está influenciada por factores socioculturales que repercuten sobre la constitución factorial final. Pese a ello, el modelo encontrado teóricamente se ajusta con la clasificación del apoyo social percibido de tipo funcional en apoyo emocional, apoyo instrumental y apoyo informacional (Barrón, 1996; Schaefer et al., 1981). 
Prosiguiendo con el análisis, se aprecia que la agrupación de los ítems a través de la rotación Promax difiere con respecto a los estudios hispanoamericanos. Para Costa et al. (2007), el factor 1 estaba conformado por lo ítems 3, 4, 7, 8, 9, 11, $13,14,16,17,18$ y 19; y para Revilla et al. (2005), el mismo factor agrupó los ítems 3, 4, 8, 9, 11, 13, 14, 16, 17 y 19; a pesar de ser muestras clínicas (enfermedad crónica), los resultados varían en el número de ítems. En el presente estudio se conservaron los ítems 3, 4, 8, 9, 13, 14, 16, 17 y 19 para el factor 1 (apoyo informacional). El ítem 14 ("Alguien con quien hacer cosas que le sirvan para olvidar los problemas”) denota para los pacientes crónicos un apoyo informacional de subtipo afectivo donde la compañía de alguien provee de información empática al paciente; es considerado por Sherbourne y Stewart (1991) como parte de la dimensión de interacción positiva.

De otro lado, el factor 2 (apoyo emocional) quedó definido por los ítems 6, 10, 11, 12, 15 y 20. En contraste con el estudio de Costa et al. (2007), que encontró los ítems 6, 10 y 20; mientras que para Revilla et al. (2005) fueron los ítems 6, $7,10,18$ y 20 . Es decir, en este factor existe una mayor discrepancia, específicamente, en los ítems 11 ("Alguien con quien pueda relajarse"), 12 ("Alguien que le prepare comida si no puede hacerlo"), y 15 ("Alguien que le ayude con las tareas domésticas"); estos dos últimos conformaban parte del apoyo instrumental en el estudio de Sherbourne y Stewart (1991). Esto se debe -en estos dos ítems- a la percepción de la ayuda más como una expresión de afecto en sí mismo que como una ayuda a sus limitaciones actuales. Y respecto al factor 3 (apoyo instrumental), los ítems fueron: 2, 5, 7 y 18. Costa et al. (2007) consideraron dentro de este factor los ítems 2, 5, 12 y 15; de igual forma, Revilla et al. (2005) encontraron los mismos ítems (2, 5,12 y 15). En el presente estudio, los ítems 7 ("Alguien con quien pasar un buen rato") y 18 ("Alguien con quien divertirse") son percibidos por el paciente con cáncer como un apoyo instrumental más que como una muestra de afecto; sin embargo, conviene analizar con un estudio más amplio esta agrupación debido a la baja consistencia con los dos estudios previos.

Se analizó la correlación entre el apoyo social percibido estructural con el apoyo social funcional y sus dimensiones. Se detectó un nivel medio positivo (.231-.498), pero en la literatura no se refiere un similar análisis previo; sin embargo, Costa et al. (2007) explicita una relación directa entre ambos tipos de apoyo social percibido (estructural y funcional). Lo mencionado también es sustentado con López y Chacón (1999) y Riquelme (1997), quienes señalan la relación de ambos tipos de apoyo social sobre el proceso salud-enfermedad.

Después de determinar los factores y sus respectivos ítems, se halló la fiabilidad de los primeros, cuyos valores oscilaron entre .708 y .908. Este hallazgo se aproxima al estudio de Sherbourne y Stewart (1991), donde lograron niveles elevados de alfa de Cronbach (.91-.96); ello, en parte, se debe a que el análisis del estudio original comprendió una muestra más significativa (2987 pacientes con enfermedad crónica). Los resultados coindicen con estudios de muestras inferiores a 500 casos como Costa et al. (2007), quienes encuentran valores entre .75 y .94; 
Revilla et al. (2005), .87 a .94; Rodríguez y Enrique (2007), .80 a .87; Alonso et al. (2007), .87 a .96; Londoño et al. (2012), .74 a .92. Dado que el apoyo social percibido es un constructo muy útil en la evaluación e intervención del paciente con cáncer (Nieto Munuera et al., 2004), contar con un instrumento con niveles elevados de confiabilidad en nuestro medio será de gran utilidad.Cabe destacar que el instrumento se enfoca específicamente a pacientes con diagnóstico de cáncer, lo cual limita su uso a dicha población.

\section{CONCLUSIONES}

1. El Cuestionario MOS de Apoyo Social Percibido presenta un nivel elevado de confiabilidad.

2. Posee un adecuado nivel de validez, quedando explicado satisfactoriamente el constructo apoyo social percibido de tipo funcional.

3. El constructo, apoyo social percibido, se agrupa en dos tipos: funcional y estructural. El primero queda definido a través de los factores: informacional, afectivo e instrumental.

\section{REFERENCIAS}

Aliaga, J., Rodríguez, L., Ponce, C., Frisancho, A., \& Enríquez, J. (2006). Escala de Desesperanza de Beck (BHS): Adaptación y Características Psicométricas. Revista de Investigación en Psicología, 9, 69-79.

Alonso, A., Montes, A., Menendez, C., \& Graça, C. (2007). Adaptação cultural e validação da versão portuguesa Questionário Medical Outcomes Study Social Support Survey (MOS-SSS). Revista Acta Médica Portuguesa, 20, 525-533.

Cea, M. (2004). Análisis multivariable. Teoría y práctica en la investigación social. Madrid: Editorial Síntesis.

Celina, H. \& Campo, A. (2005). Aproximación al uso del coeficiente alfa de Cronbach. Revista Colombiana de Psiquiatría, 34, 572-580.

Costa, G., Salamero, M., \& Gil, Fr. (2007). Validación del cuestionario MOS-SSS de apoyo social en pacientes con cáncer. Revista Médica Clínica de Barcelona, $128,687-691$.

Costello, A.B. \& Osborne, J.W. (2005). Best practices in exploratory factor analysis: Four recommendations for getting the most from your analysis. Practical Assessment Research \& Evaluation, 10, 1-9.

Cruzado, J. (2010). Tratamiento psicológico en pacientes con cáncer. Madrid: Síntesis. 
Fernández, R. (2005). Redes sociales, apoyo social y salud. Recuperado de: http:// antropologia.uab.es/Periferia/english/number3/periferia_3_3.pdf

Kerlinger, Fr. \& Lee, H. (2002). Investigación del Comportamiento (4a Ed.). México: McGraw-Hill Interamericana.

Hogan, Th. (2004). Pruebas psicológicas. México: Manual Moderno.

López, M. \& Chacón, F. (1999). Intervención Psicosocial y Servicios Sociales: Un enfoque participativo. Madrid: Editorial Síntesis.

Merino, C., Pflucker, D. \& Riaño-Hernández, D. (2012). Análisis factorial exploratorio del Inventario de Depresión Estado-Rasgo (ST-DEP) en adolescentes. Revista Diversitas - Perspectivas en Psicología, 8, 319-330.

Montero, I. \& Leon, G. (2007). A guide for naming research studies in Psychology. International Journal of Clinical and Health Psychology, 7, 847-862.

Nieto Munuera, J., Abad Mateo, M.A., Esteban A.M., \&Tejeira M. (2004). Psicología para Ciencias de la Salud: Estudio del comportamiento humano para la enfermedad. España: McGraw-Hill Interamericana.

Nolan, S. \& Heinzen, Th. (2011). Statistics for the Behavioral Sciences. USA: Worth Publishers.

Reise, S.P., Waller, N.G. \& Comrey, A.L. (2000). Factor analysis and scale revision. Psychological Assessment, 12, 287-297.

Revilla, L., Luna, J., Bailón, E., \& Medina, I. (2005). Validación del cuestionario MOS de apoyo social en Atención Primaria. Revista Medicina de Familia, $6,10-18$.

Riquelme, A. (1997). Depresión en residencias geriátricas: Un estudio empírico. Murcia: Servicio de Publicaciones de la Universidad de Murcia

Rodríguez, S. \& Enrique, H. (2007). Validación argentina del cuestionario MOS de Apoyo Social Percibido. Revista Psicología, Cultura y Sociedad, 7, 155-168.

Rodríguez, J. \& Neipp, M. (2008). Manual de Psicología Social de la Salud. Madrid: Síntesis.

Rodríguez, S. (2011). Adaptación a Niños del cuestionario MOS de Apoyo Social Percibido. Revista Liberabit, 17, 17-128.

Schaefer, C., Coyne, J. C. \& Lazarus, R. S. (1981). The Health Related Functions of Social Support. Journal of Behavioral Medicine, 4(4), 381-406.

Sherbourne, C. \& Stewart, A. (1991). The MOS Social Support Survey. Recuperado de: http://cmcd.sph.umich.edu/assets/files/Repository/Women\%20Take\%20 Pride/The\%20MOS\%20Social\%20Support\%20Survey.pdf 
Terol, M., López, S., Neipp, M., Rodríguez, J., Pastor, M., \& Martin-Aragon, M. (2004). Apoyo social e instrumentos de evaluación: revisión y clasificación. Anuario de Psicología, 35, 23-45.

Welch, S., \& Comer, J. (1988). Quantitative methods for public administration: Techniques and applications. Chicago: Dorsey Press.

\section{ANEXOS}

\section{Anexo 1}

Tabla 5. Normalidad de los ítems del Cuestionario MOS de Apoyo Social de Sherbourne y

Stewart $(n=100)$

\begin{tabular}{|c|c|c|c|c|}
\hline \multirow{2}{*}{ Ítem } & \multicolumn{2}{|c|}{ Parámetros } & \multirow{2}{*}{ Kolmogorov-Smirnov Z } & \multirow{2}{*}{ Sig. (bilateral } \\
\hline & Media & $\mathrm{DE}$ & & \\
\hline Ítem 2 & 4.35 & 0.88 & 3.398 & 0.000 \\
\hline Ítem 3 & 4.21 & 0.86 & 2.619 & 0.000 \\
\hline Ítem 4 & 4.11 & 0.95 & 2.450 & 0.000 \\
\hline Ítem 5 & 4.31 & 0.85 & 2.918 & 0.000 \\
\hline Ítem 6 & 4.50 & 0.76 & 3.850 & 0.000 \\
\hline Ítem 7 & 4.30 & 0.86 & 3.025 & 0.000 \\
\hline Ítem 8 & 4.16 & 0.91 & 2.628 & 0.000 \\
\hline Ítem 9 & 4.05 & 0.93 & 2.485 & 0.000 \\
\hline Ítem 10 & 4.41 & 0.85 & 3.452 & 0.000 \\
\hline Ítem 11 & 4.34 & 0.86 & 3.398 & 0.000 \\
\hline Ítem 12 & 4.44 & 0.78 & 3.629 & 0.000 \\
\hline Ítem 13 & 4.17 & 0.90 & 2.519 & 0.000 \\
\hline Ítem 14 & 4.15 & 0.88 & 2.528 & 0.000 \\
\hline Ítem 15 & 4.29 & 0.87 & 3.133 & 0.000 \\
\hline Ítem 16 & 3.96 & 0.93 & 2.080 & 0.000 \\
\hline Ítem 17 & 3.97 & 0.94 & 2.328 & 0.000 \\
\hline Ítem 18 & 4.25 & 0.83 & 2.659 & 0.000 \\
\hline Ítem 19 & 4.08 & 0.86 & 2.230 & 0.000 \\
\hline Ítem 20 & 4.58 & 0.70 & 3.961 & 0.000 \\
\hline
\end{tabular}

Nota: $\mathrm{DE}=$ Desviación estándar 


\section{Anexo 2}

Cuestionario MOS de Apoyo Social Percibido

\begin{tabular}{|c|c|c|c|c|c|c|}
\hline & Escriba el número aproximado de amigos cercanos y & nilia & cerca & & & \\
\hline & $\begin{array}{l}\text { Iarca con una } \mathrm{X} \text { en el cuadrito que mejor se acerque } \\
\qquad \text { a su respuesta }\end{array}$ & 异 & $\begin{array}{l}\tilde{J} \\
\tilde{\Xi} \\
z \\
\tilde{\tilde{J}} \\
\tilde{J}\end{array}$ & 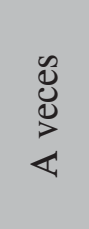 & 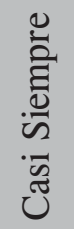 & 营 \\
\hline 2 & $\begin{array}{l}\text { Alguien que le ayude cuando tenga que estar en } \\
\text { cama }\end{array}$ & $\mathrm{N}$ & $\mathrm{CN}$ & $\mathrm{AV}$ & $\mathrm{CS}$ & $\mathrm{S}$ \\
\hline 3 & $\begin{array}{l}\text { Alguien con quien pueda contar cuando necesite } \\
\text { hablar }\end{array}$ & $\mathrm{N}$ & $\mathrm{CN}$ & AV & $\mathrm{CS}$ & $\mathrm{S}$ \\
\hline 4 & Alguien que le aconseje cuando tenga problemas & $\mathrm{N}$ & $\mathrm{CN}$ & $\mathrm{AV}$ & $\mathrm{CS}$ & $\mathrm{S}$ \\
\hline 5 & Alguien que le lleve al médico cuando lo necesite & $\mathrm{N}$ & $\mathrm{CN}$ & AV & $\mathrm{CS}$ & $\mathrm{S}$ \\
\hline 6 & Alguien que le muestre amor y afecto & $\mathrm{N}$ & $\mathrm{CN}$ & $\mathrm{AV}$ & $\mathrm{CS}$ & $\mathrm{S}$ \\
\hline 7 & Alguien con quien pasar un buen rato & $\mathrm{N}$ & $\mathrm{CN}$ & AV & $\mathrm{CS}$ & $\mathrm{S}$ \\
\hline 8 & $\begin{array}{l}\text { Alguien que le informe y le ayude a entender la } \\
\text { situación }\end{array}$ & $\mathrm{N}$ & $\mathrm{CN}$ & AV & $\mathrm{CS}$ & $\mathrm{S}$ \\
\hline 9 & $\begin{array}{l}\text { Alguien en quien confiar o con quien hablar de sí } \\
\text { mismo y sus preocupaciones }\end{array}$ & $\mathrm{N}$ & $\mathrm{CN}$ & AV & $\mathrm{CS}$ & $\mathrm{S}$ \\
\hline 10 & Alguien que le abrace & $\mathrm{N}$ & $\mathrm{CN}$ & AV & CS & S \\
\hline 11 & Alguien con quien pueda relajarse & $\mathrm{N}$ & $\mathrm{CN}$ & AV & $\mathrm{CS}$ & $\mathrm{S}$ \\
\hline 12 & Alguien que le prepare comida si no puede hacerlo & $\mathrm{N}$ & $\mathrm{CN}$ & AV & $\mathrm{CS}$ & S \\
\hline 13 & Alguien cuyo consejo realmente desee & $\mathrm{N}$ & $\mathrm{CN}$ & $\mathrm{AV}$ & $\mathrm{CS}$ & $\mathrm{S}$ \\
\hline 14 & $\begin{array}{l}\text { Alguien con quien hacer cosas que le sirvan para } \\
\text { olvidar los problemas }\end{array}$ & $\mathrm{N}$ & $\mathrm{CN}$ & AV & $\mathrm{CS}$ & $\mathrm{S}$ \\
\hline 15 & Alguien que le ayude con las tareas domésticas & $\mathrm{N}$ & $\mathrm{CN}$ & AV & $\mathrm{CS}$ & S \\
\hline 16 & $\begin{array}{l}\text { Alguien con quien compartir sus temores y } \\
\text { problemas íntimos }\end{array}$ & $\mathrm{N}$ & $\mathrm{CN}$ & $\mathrm{AV}$ & $\mathrm{CS}$ & $\mathrm{S}$ \\
\hline 17 & $\begin{array}{l}\text { Alguien que le aconseje cómo resolver sus } \\
\text { problemas personales }\end{array}$ & $\mathrm{N}$ & $\mathrm{CN}$ & AV & $\mathrm{CS}$ & $\mathrm{S}$ \\
\hline 18 & Alguien con quien divertirse & $\mathrm{N}$ & $\mathrm{CN}$ & AV & $\mathrm{CS}$ & $\mathrm{S}$ \\
\hline 19 & Alguien que comprenda sus problemas & $\mathrm{N}$ & $\mathrm{CN}$ & AV & $\mathrm{CS}$ & $\mathrm{S}$ \\
\hline 20 & Alguien a quien amar y sentirse querido & $\mathrm{N}$ & $\mathrm{CN}$ & AV & $\mathrm{CS}$ & $\mathrm{S}$ \\
\hline
\end{tabular}

